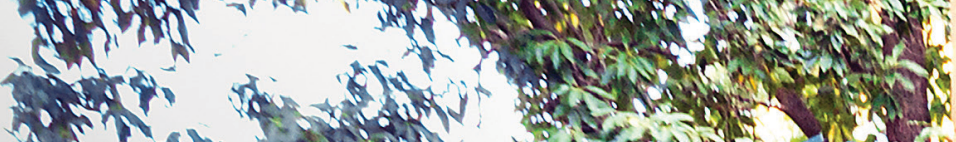

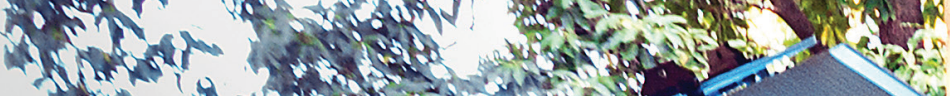

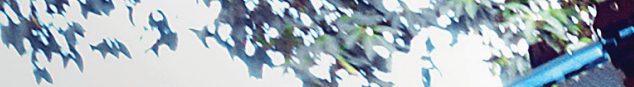

(a)

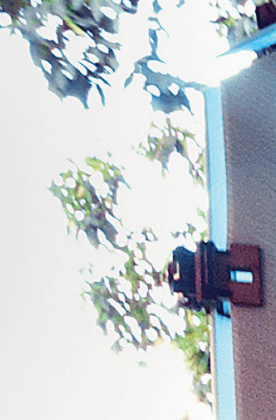

20

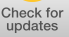

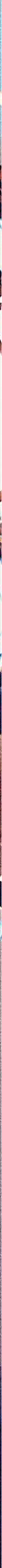




\begin{tabular}{l}
\hline 8 DEMO HOUR \\
12 WHAT ARE YOU READING? \\
14 HOW WAS IT MADE? \\
\hline 16 DAY IN THE LAB \\
\hline
\end{tabular}

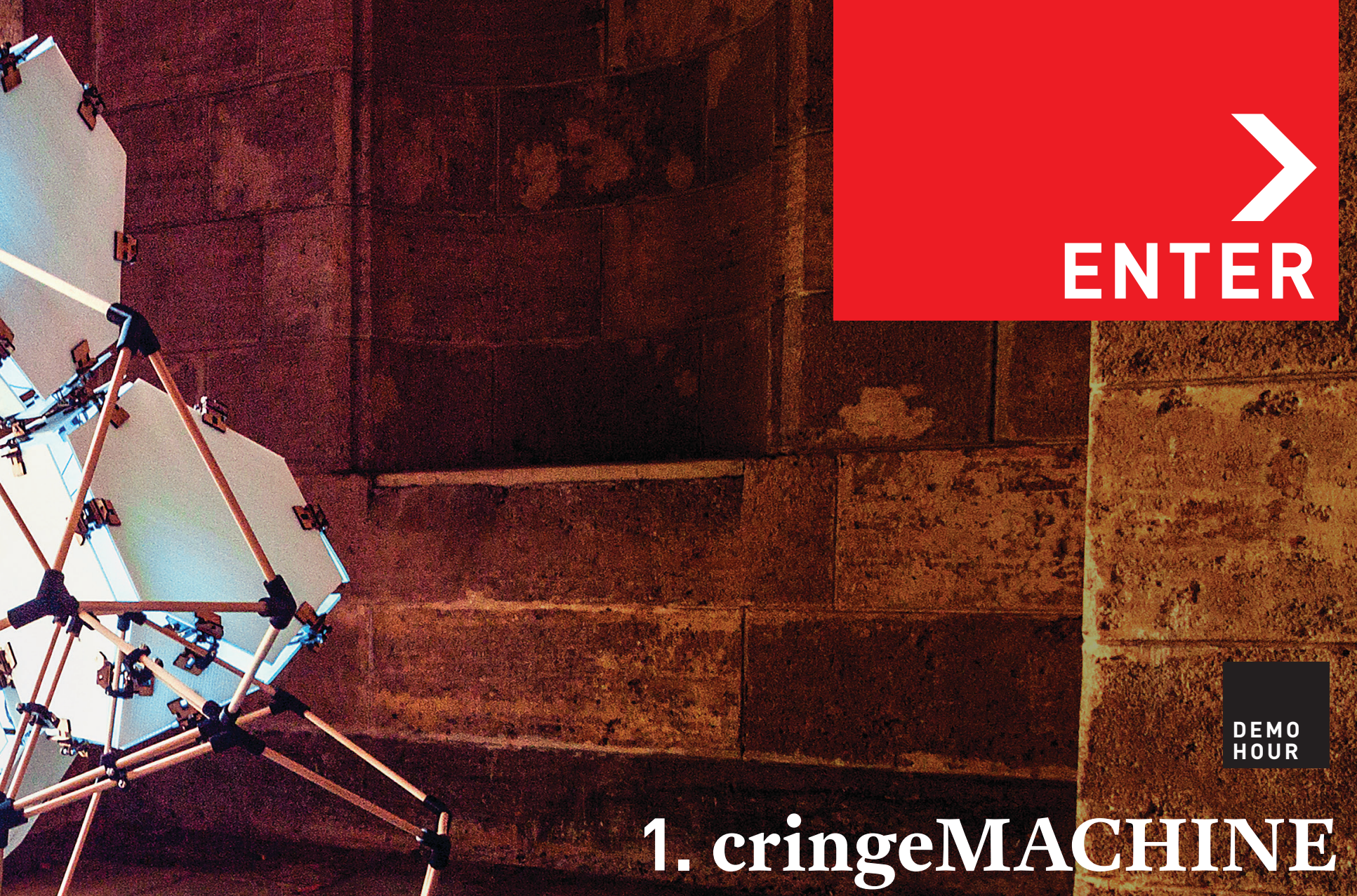

cringeMachine was an early prototype for an interactive public installation.

The purpose of the project was to develop a sculpturat, crystalline-like aggregate form that could be used to experiment with modular interactive systems and study their effect on public space. We also wanted to explore people's reactions to its rudimentary agency. The sculpture incorporated a speaker, a sound sensori and a simple Arduino circuit connected to linear array of LED lights, which reacted to sound levels around it in real time. This installation is evocative of systems in nature that develop from simple rules and behaviors to create ah effect that is greater than the sum of its parts.

nttp://news.curtin.edu.au/stories/forays-into-interactive-architecture/ n http://www.thedeadpixelproject.com/subpages/research/cringeMACHINE.html fo.

- https://vimeo.com/185271747

Robert Cameron, University of Western Austratia $\rightarrow$ robert.cameronaresearch.uwa.edu.au

Andrei Smolik, Curtin University $\rightarrow$ andrei.smolik1@ curtin.edu.au 

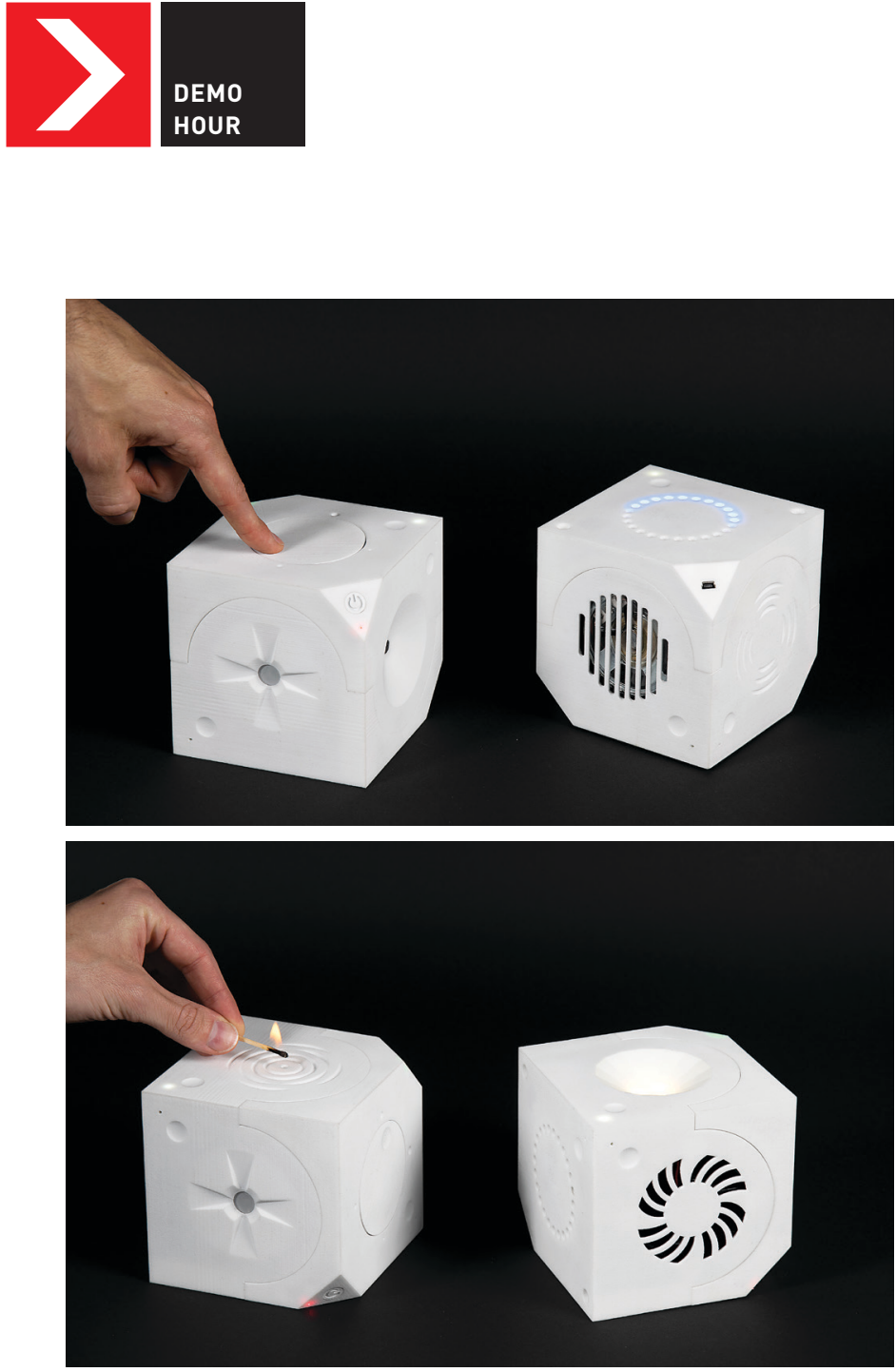

2

The sensor on the top side of one cube communicates wirelessly with the actuator on the top side of the other cube.

\section{Loaded Dice}

Loaded Dice is a co-design tool used to explore the design space of smart connected things. The tool consists of two Arduinobased 3D-printed cubes. One of the devices is equipped with a different sensor on each of its six sides, while the other is equipped with a different actuator on each side. Whichever side is on top is active, communicating wirelessly over distance with the top side of the other cube. Loaded Dice supports creativity, imagination, and exploration in the design process of smart and multisensory devices and services, especially in the early ideation stages.

Lefeuvre, K., Totzauer, S., Bischof, A. Kurze, A., Storz, M., Ullmann, L., and Berger, A. Loaded Dice: Exploring the design space of connected devices with blind and visually impaired people. Proc. of the 9th Nordic Conference on Human-Computer Interaction. ACM, New York, 2016, Article No. 31. (1) Lefeuvre, K., Berger, A., Kurze, A., Totzauer, S., Storz, M., and Bischof, A. Smart connected sensations: $\mathrm{Co}$-creating smart connected applications through distributed serendipity. Proc. of the 9th Nordic Conference on HumanComputer Interaction. ACM, New York, 2016, Article No. 88. () http://nebeneinandermiteinander.de/

Kevin Lefeuvre, Albrecht Kurze, Sören Totzauer, Michael Storz, Andreas Bischof, and Arne Berger, Technische Universität Chemnitz $\rightarrow$ kevin.lefeuvrela

informatik.tu-chemnitz.de

\section{Zooids}

Swarm user interfaces are a new class of human-computer interface comprising many autonomous robots that handle both display and interaction. Zooids is an open-source, open-hardware platform for developing tabletop swarm interfaces. The platform consists of a collection of custom-designed wheeled micro robots, each $2.6 \mathrm{~cm}$ in diameter, a radio base station, a high-speed digital-light processing (DLP) structured light projector for optical tracking, and a software framework for application development and control. We 

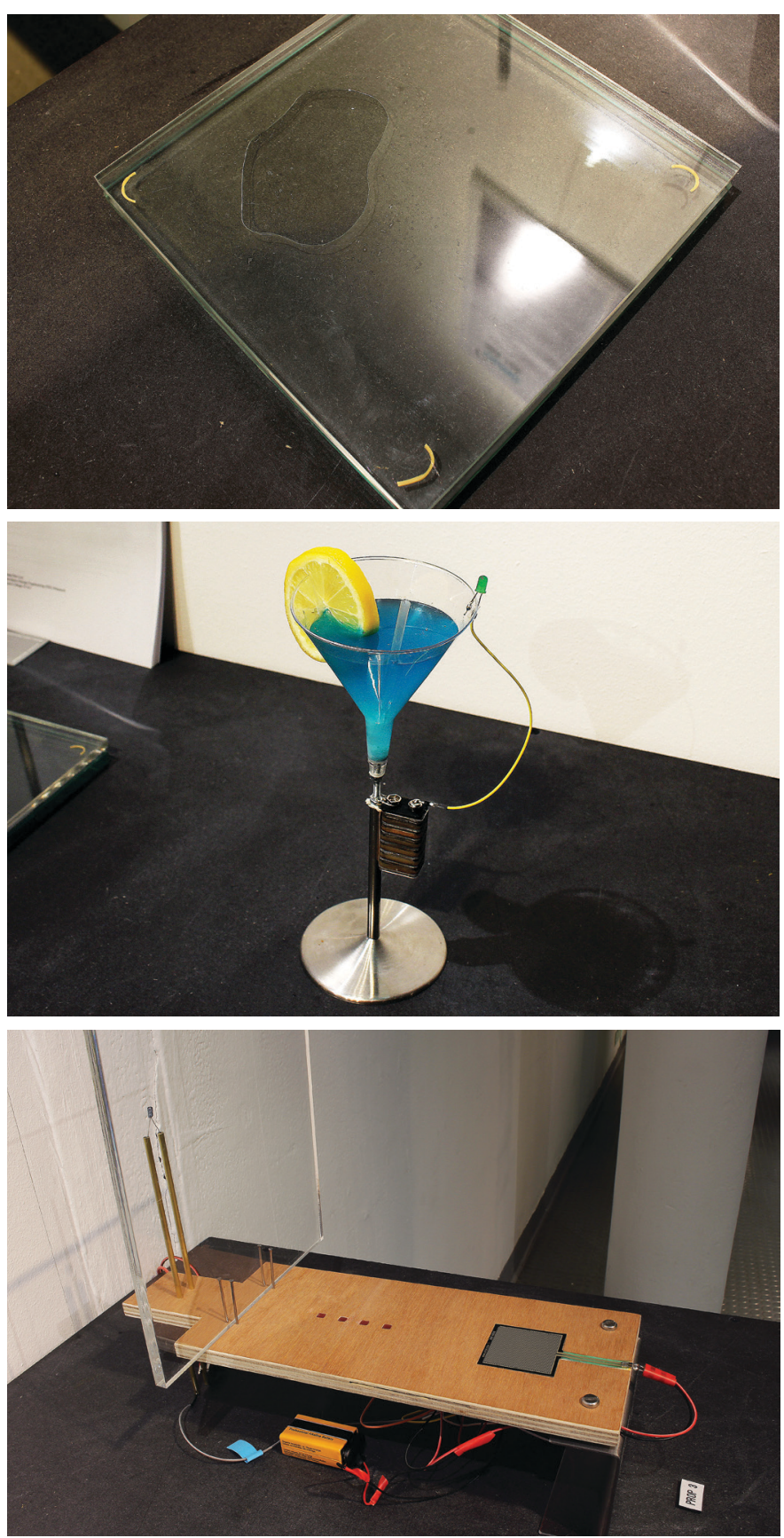

have explored the potential of tabletop swarm user interfaces through a set of application scenarios developed with Zooids and proposed general design considerations unique to swarm user interfaces.

Le Goc, M. et al. Zooids:

Building blocks for swarm user interfaces. Proc. of the 29th Annual ACM Symposium on User Interface Software \& Technology. ACM, New York, 2016, 97-109.

() http://www.aviz.fr/swarmui () http://shape.stanford.edu/ research/swarm/

(1) https://vimeo.com/191648523
Mathieu Le Goc, Inria and Université Paris-Sud $\rightarrow$ mathieu.le-gocQinria.fr Lawrence H. Kim,

Stanford University

$\rightarrow$ lawkimastanford.edu Ali Parsaei, Stanford University $\rightarrow$ aparsaeidalumni.stanford.edu Jean-Daniel Fekete, Inria

$\rightarrow$ jean-daniel-feketedinria.fr

Pierre Dragicevic, Inria

$\rightarrow$ pierre.dragicevicainria.fr

Sean Follmer, Stanford University $\rightarrow$ sfollmerdastanford.edu

\section{Three Studies of Synaesthesia} is a research project that attempts to explore some of the intriguing properties of synaesthesia. Three props highlight some provocative elements of synaesthesia that can be used within a design context. Prop 1 tries to illuminate the transformative properties of synaesthesia. Prop 2 explores real and surreal images of taste. Prop 3 mimics a narrative of
Three Studies of Synaesthesia

4

Prop 1 considers how weight and scale could be associated with each other. The liquid between the two glass sheets immediately expands its surface according to the pressure it receives on the glass surface.

4

Prop 2 is an attempt to question the difference between our mental images and the real images of taste. How does electricity taste in your mind? What does it really taste like?

4

Prop 3 mimics the narratives of anger-related synaesthesia and its level of intensity. When a user exerts force (anger) on the pad, the prop will make a noise and turn on the red LEDs proportionately to the amount of pressure received, indicating the amount of anger.

anger-related synaesthesia. The peculiar properties of synaesthesia provide a speculative domain where people can explore potential interactive features within the context of design.

http://www.changheelee.com/ three-studies-of-synaesthesia.html

https://www.youtube.com/ watch?v=zbq37Cc $-y 04$

Chang Hee Lee, Royal College of Art

$\rightarrow$ changhee.leelanetwork.rca.ac.uk 